\title{
CARACTERIZAÇÃO FÍSICO-QUÍMICA DE FRUTOS NATIVOS DA REGIÃO SUL DO BRASIL
}

\author{
Souza AG, ${ }^{*}$ Fassina $A C,{ }^{\dagger}$ Saraiva FRS, ${ }^{\ddagger}$ Souza $L^{\S}$
}

\section{Resumo}

Os frutos nativos vêm recebendo atenção especial, não apenas por seu uso in natura, mas também pela forma como são processados. Nesse sentido, o objetivo com este trabalho foi avaliar atributos físico-químicos de sólidos solúveis (SS), acidez titulável (AT), relação SS/AT, pH e conteúdo de vitamina $C$ em frutos de açaí (Euterpe edulis), araçá amarelo e vermelho (Psidium cattleianum), butiá (Butia capitata), guabiroba (Campomanesia xanthocarpa), jabuticaba (Plinia cauliflora) e pitanga (Eugenia uniflora) colhidos no ponto ideal de consumo. Os frutos foram processados e as análises realizadas no laboratório de Fisiologia e Pós-colheita do Instituto Federal Catarinense (IFC) de Rio do Sul. Os teores de AT foram obtidos por titulometria, os SS, por refratômetria digital, a relação entre SS/AT foi calculada pela divisão dos SS, pela AT, o pH foi determinado com pHmetro de bancada em suco extraído dos frutos, e o conteúdo de vitamina $C$ foi determinado pelo método

\footnotetext{
* Doutora em Produção Vegetal pela Universidade do Estado de Santa Catarina; Mestre em Manejo do Solo pela Universidade do Estado de Santa Catarina; Professora no Instituto Federal Catarinense; alexandra.souza@ifc.edu.br

† Graduanda em Engenharia Agronômica pelo Instituto Federal Catarinense; ana_fassina@hotmail.com

‡ Mestre em Ciências Farmacêuticas pela Universidade do Vale do Itajaí; Graduada em farmácia e bioquímica pela Universidade Católica de Pelotas; Professora no Instituto Federal Catarinense; fatima.saraiva@ifc.edu.br

§ Mestre em Desenvolvimento Regional pela Universidade Regional de Blumenau; Especialista em Pedagogia Gestora pela Faculdade de Educação de Joinville; Supervisor Educacional no Instituto Federal Catarinense; laercio.souza@ifc.edu.br
} 
espectrofotométrico, utilizando-se 2,4-denitrofenilhidrazina. Frutos de guabiroba, açaí, butiá e jabuticaba apresentaram os maiores valores de AT e, junto com a pitanga, os maiores valores de SS. A jabuticaba apresentou maior $\mathrm{pH}$, e o butiá e a pitanga os menores. O conteúdo médio de vitamina $C$ foi de $56,14 \mathrm{mg}_{100 \mathrm{~g}^{-1}}$ de massa fresca (MF), indicando que todos os frutos estudados são importantes fontes

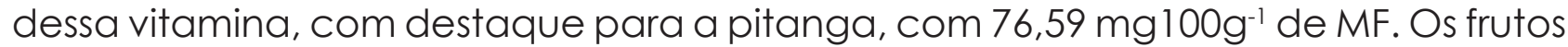
apresentam características físico-químicas adequadas tanto para o consumo in natura quanto na forma processada.

Palavras-chave: Sólidos solúveis. Acidez titulável. PH. Vitamina C.

\section{Physicochemical characterization of south brazialian native fruits}

\section{Abstract}

The native fruits have receiving special attention, not only for their in natura use but also in the processed form. The objective of this work was to evaluated the physicochemical attributes soluble solids (SS), titratable acidity (AT), SS/AT ratio, $\mathrm{pH}$ and vitamin C contents in açaí (Euterpe edulis), yellow and red araçá (Psidium cattleianum), butiá (Butia capitata), guabiroba (Campomanesia xanthocarpa), jabuticaba (Plinia cauliflora) and pitanga (Eugenia uniflora) fruits at harvested. The fruits were processed and analyzes in the Laboratory of Physiology and Postharvest of the Instituto Federal Catarinense (IFC) of Rio do Sul. The levels of AT were obtained by titrations, the SS by digital refractometry, the ratio (SS/AT) was calculated by dividing the SS by AT, the $\mathrm{pH}$ was determined with a pHmeter in juice extracted from the fruits and the vitamin $\mathrm{C}$ content by the spectrophotometric method, using 2,4-denitrophenylhydrazine. Fruits of guabiroba, açaí, butiá and jabuticaba presented the highest values of AT and, together with pitanga, the highest values of SS average contents of $1.40 \%$ and $9.05 \%$, respectively. The highest $\mathrm{pH}$ were observed in jabuticaba and the lowest in butiá and pitanga. The mean content of

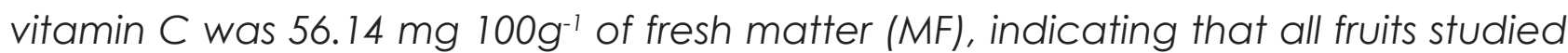
are important sources of this vitamin, especially pitanga, with $76.59 \mathrm{mg} 100 \mathrm{~g}-1$ of MF. The fruits present physicochemical characteristics suitable for in natura and processed form consumption.

Keywords: Soluble solids. Titratable acidity. pH. Vitamin C.

\section{INTRODUÇÃO}

Recentemente, o interesse no consumo de frutos nativos vem crescendo, especialmente dos pouco conhecidos, também chamados de frutos não tradicionais, em razão do potencial benéfico à saúde humana que apresentam, ${ }^{1-4}$ 
atribuído à presença de substâncias antioxidantes como os compostos fenólicos, as vitaminas e os minerais que contribuem na prevenção de muitas doenças. ${ }^{5,6}$ Muitos frutos, como mirtilo, jabuticaba e uva, de coloração mais escura, estão sendo chamados de super frutos principalmente pela indústria de alimentos, que vem apostando nos ingredientes funcionais na elaboração de seus produtos para atender à tendência global de consumo de alimentos naturais. ${ }^{3}$

Nesse contexto estão inseridos frutos como araçá amarelo e vermelho (Psidium cattleianum), jabuticaba (Plinia cauliflora), pitanga (Eugenia uniflora), butiá (Butia capitata), guabiroba (Campomanesia xanthocarpa) e açaí (Euterpe edulis) (também conhecido como juçara), todos nativos da flora brasileira e recentemente considerados com potencial econômico para o futuro na região Sul do Brasil.?

O araçá, a jabuticaba, a pitanga e a guabiroba são frutíferas pertencentes a família das Myrtaceae. ${ }^{4,7} \mathrm{O}$ araçazeiro é uma arvoreta de até seis metros de altura de flores brancas e fruto do tipo baga globosa de coloração amarela ou vermelha quando madura. A polpa pode ser branca, amarelo-clara ou vermelha, suculenta e de sabor doce, levemente acidulado e adstringente, e é tradicionalmente consumida in natura.?

A jabuticaba é uma planta muito popular no Brasil, e sua árvore pode chegar até 15 metros de altura. As flores, de coloração branca nascem diretamente no tronco e nos galhos da árvore. Os frutos são globosos e quando maduros apresentam coloração negra brilhante e polpa branca de sabor inigualável, tradicionalmente consumidos in natura, porém podem ser utilizados na fabricação de sucos, vinhos, geleias, doces e licores, com potencial para uso em pratos da alta gastronomia, como molhos agridoces e sobremesas. ${ }^{7}$

A pitangueira é um arbusto de dois a quatro metros de altura, ramificada e de copa arredondada, muito empregada no paisagismo. As flores são solitárias ou fasciculadas de coloração creme, o fruto é uma baga globosa, deprimida nos polos, com sulcos marcados no sentido longitudinal, de coloração laranjada, vermelha, vermelho-escura, podendo chegar a quase negro quando maduro. Seu principal consumo é in natura e na forma de polpa congelada para suco.?

A guabirobeira pode chegar a 15 metros de altura, suas flores são de coloração creme e o fruto é do tipo baga amarela quando maduro. A polpa é suculenta, esbranquiçada, doce, aromática, envolvendo inúmeras sementes. No Brasil, predomina o consumo in natura do fruto, no entanto o emprego no preparo de polpa congelada para suco e outras bebidas, como licores, é promissor em razão do seu aroma e sabor característico.?

Frutos de açaí (Euterpe edulis), também conhecido como palmito juçara, são originários de palmeira pertencente à família das Arecaceae. ${ }^{4} \mathrm{O}$ fruto é redondo e de coloração púrpura escura quando maduro, atribuída à presença de elevados conteúdos de antocianina, similar ao fruto de açaí (Euterpe oleraeae).? 
O fruto é consumido principalmente na forma de polpa congelada para suco, preparo de bebidas energéticas e sobremesas. Considerado importante fonte de energia, é reportado que a polpa do fruto é rica em antocianinas e carotenoides, apresentando grande potencial antioxidante. ${ }^{2}$

O butiazeiro é uma palmeira de seis a sete metros de altura, muito empregada no paisagismo urbano, em razão de seu porte mediano coloração azul-esverdeada das folhas. Os frutos são globosos, de sabor doce-acidulado, coloração amarela quando maduros e distribuídos em grandes cachos. A polpa fortemente aromática é apreciada principalmente in natura e na produção de polpa congelada para sucos, além de geleias, licores, vinhos, doces, entre outros.?

Para algumas espécies já existe algum tipo de uso ou mercado estabelecido, mas apenas em âmbito local ou regional. ${ }^{7.8}$ As perspectivas promissoras para a exploração de frutos nativos pouco conhecidos se devem, entre outros, aos sabores especiais e diversificados e aos elevados teores de açúcares, vitaminas e minerais, que promovem grande aceitação popular. 9

Muitos desses frutos são altamente perecíveis, sendo este um dos fatores que limitam a comercialização in natura. Assim, a elaboração de produtos como polpa congelada para sucos, doces, geleias, sorvetes e licores, além do seu emprego na fabricação de cosméticos e alimentos funcionais, ${ }^{10,11}$ apresenta-se como uma alternativa para o aproveitamento desses frutos na época da safra, evitando o desperdício e agregando valor aos frutos regionais.

Nos últimos anos, o número de publicações sobre algumas espécies frutíferas nativas tem aumentado consideravelmente, ${ }^{3,5,12}$ no entanto, para a grande maioria, dados sobre a composição física, química e nutricional ainda são escassos. 0 conhecimento desses atributos, entre outros, pode contribuir para a expansão do consumo e, consequentemente, da produção, constituindo-se em uma alternativa para a agricultura familiar na região Sul do Brasil, que tem na fruticultura uma forma de diversificação da propriedade e de fixação do homem no campo, por se tratar de uma atividade exigente em mão de obra, permitindo a oferta de novas opções de sabores e aromas, ${ }^{13}$ além de frutos com propriedades bioativas desejáveis em razão da presença de minerais, vitaminas, compostos fenólicos e ação antioxidante. ${ }^{1,2,4,6}$

O objetivo neste estudo é caracterizar, a partir de atributos físicos e químicos, os frutos de diferentes espécies frutíferas nativas do Brasil (araçá amarelo e vermelho, pitanga, jabuticaba, guabiroba, butiá e açaí) colhidos no ponto de consumo.

\section{MATERIAL E MÉTODOS}

Frutos das diferentes espécies nativas foram colhidos de no mínimo três plantas (de cada espécie) de ocorrência em áreas no Instituto Federal Catarinense (IFC) 
de Rio do Sul (latitude $27^{\circ} 12^{\prime} 51$ " Sul, longitude 49³8'35" Oeste e altitude de 698 metros), pertencente ao bioma Mata Atlântica, no ponto de colheita comercial, e foram imediatamente transportados ao laboratório de Fisiologia Vegetal e Póscolheita do IFC para realização das análises.

As sementes dos frutos de butiá, jabuticaba, pitanga e açaí, após a colheita, foram retiradas manualmente, e a polpa processada com auxílio de um mixer. Os frutos de guabiroba, araçá vermelho e amarelo foram processados inteiros.

$\mathrm{Na}$ polpa dos frutos foram avaliados os atributos de acidez titulável (AT), teores de sólidos solúveis (SS), relação SS/AT, pH e conteúdos de vitamina C (ácido ascórbico).

Os teores de AT foram obtidos por titulometria de $10 \mathrm{~mL}$ do suco dos frutos (processada com auxílio de um mixer) diluídos em $90 \mathrm{~mL}$ de água destilada com hidróxido de sódio $0,1 \mathrm{~N}$ até $\mathrm{pH} 8,1$ ), e os resultados foram expressos em \% de ácido cítrico. Os teores SS foram determinados em refratômetro digital com compensação automática de temperatura em suco extraído conforme descrito para AT. A relação entre SS/AT foi calculada pela divisão dos SS pela AT. O pH foi determinado com pHmetro de bancada (modelo mPA210) em suco extraído da planta. ${ }^{14}$

O conteúdo de vitamina $C$ foi determinado pelo método espectrofotométrico, utilizando-se 2,4-denitrofenilhidrazina. ${ }^{15}$ Foi utilizado $1 \mathrm{~g}$ da amostra, macerado em $5 \mathrm{~mL}$ de ácido oxálico (0,5\%). Após filtragem, foi tomado $1 \mathrm{~mL}$ da amostra e adicionados $3 \mathrm{~mL}$ de ácido oxálico, cinco gotas do agente oxidante 2,6 diclorofenol-indofenol (2,6-DCFI), $1 \mathrm{~mL}$ de 2,4 dinitrofenilhidrazina (2,4-DNPH) e uma gota de tiureia. Após ferver em banho-maria por 15 minutos e resfriar em banho de gelo, foram adicionados $5 \mathrm{~mL}$ de ácido sulfúrico. As leituras foram realizadas em espectrofotômetro a $520 \mathrm{~nm}$, e os resultados expressos em $\mathrm{mg}$ de ácido ascórbico por $100 \mathrm{~g}^{-1}$ de massa fresca (MF).

O delineamento estatístico utilizado foi inteiramente casualizado, com cinco repetições, cada repetição com $200 \mathrm{~g}$ de polpa, totalizando $1 \mathrm{~kg}$ de polpa para cada espécie. Os dados foram submetidos à análise de variância, e os resultados comparados pelo método de Tukey a 5\% de probabilidade utilizando o programa SASM-Agri. ${ }^{16}$

\section{RESULTADOS E DISCUSSÃO}

O conteúdo médio de SS obtido para os diferentes frutos analisados foi de $8,91 \%$. Os maiores valores foram observados em frutos de guabiroba, açaí, butiá e pitanga, seguidos de jabuticaba, araçá vermelho e amarelo (Tabela 1).

Conteúdos médios semelhantes de SS foram observados em frutos de butiá $(10,32 \%)$ coletados no Rio Grande do Sul, ${ }^{6}$ frutos de açaí $(11,6 \%)$ e de pitanga $(11,47 \%)$ coletados em São Paulo., 817 
Ainda, para butiás colhidos no Rio Grande do Sul, foram observados valores de SS variando de 11,38 a 11,98\% de acordo com a população de ocorrência. ${ }^{18}$ No entanto, para guabiroba, os valores apresentados no presente estudo são inferiores aos $13,02 \%$ e $11,55 \%$ reportados em frutos coletados de plantas do cerrado brasileiro,, 19 aos $11 \%$ em frutos maduros de araçá amarelo (P. guineense) colhidos em Goiás ${ }^{20}$ e aos 10,10\% em araçá vermelho provenientes do Estado do Paraná. ${ }^{13}$

Diferenças observadas nos teores de SS podem ser atribuídas às variações genéticas, às condições de cultivo e ao ponto de colheita. ${ }^{21}$ Teores de SS variaram de 11,6 a 17,9\% em frutos de jabuticaba colhidos em diferentes regiões do Estado de São Paulo..$^{22}$ Em araçá, os valores variaram de 8 a $11 \%$ de acordo com a classe de maturação. ${ }^{20}$ Teores elevados de SS são uma característica interessante para frutos comercializados in natura, pois o mercado prefere frutos doces.

Os valores de AT também apresentaram diferenças entre os frutos das diferentes espécies avaliadas. Os frutos de guabiroba, açaí, butiá e jabuticaba apresentaram os maiores valores de AT, enquanto os frutos de pitanga, araçá amarelo e vermelho, os menores (Tabela 1). Os valores obtidos neste trabalho são semelhantes ao $1,24 \%$ reportado em polpa de pitanga, ${ }^{8}$ ao $1,21 \%$ em frutos maduros de araçá ( $P$. guineense $)^{20}$ e aos $0,88 \%$ a $1,62 \%$ em frutos de jabuticaba 'Sabará' provenientes de diferentes regiões do Estado de São Paulo. ${ }^{22}$

Para guabiroba os valores de AT são superiores aos $0,33 \%$ e $0,183 \%$ encontrados em frutos coletados no cerrado, 9.19 assim como para araçá vermelho $(0,30 \%)$ colhido no Estado do Paraná ${ }^{13}$ e ao $1,2 \%$ em frutos maduros de açaí ( $E$. oleracea). ${ }^{23}$ Enquanto para butiá, os valores de AT obtidos são inferiores aos 2,18 a 2,59\% apresentados em frutos colhidos em diferentes populações de butiá no Rio Grande do Sul. ${ }^{18}$

A relação entre sólidos solúveis e acidez titulável (SS/AT) é considerada um critério de avaliação do flavor em frutos além de indicativo do grau de maturação. ${ }^{24}$ Na média dos sete frutos avaliados, a relação foi de 6,62, porém os frutos não apresentaram diferenças significativas nos valores da relação SS/AT (Tabela 1). Os frutos de jabuticaba apresentaram valores inferiores aos 14,66 e aos 7,43-18,98 de frutos oriundos de diferentes regiões de cultivos no Estado de São Paulo. ${ }^{25,22}$

Valores superiores também foram reportados em pitanga $(9,25), 8$ araçá vermelho $(33,66),{ }^{13}$ araçá amarelo $(9,10)$ (P. guineense) ${ }^{20}$ e guabiroba $(39,70) .{ }^{9}$ Já para butiá, foram reportados valores inferiores aos deste estudo, variando de 4,72 a 6,00 de acordo com as populações de onde foram colhidos os frutos no Rio Grande do Sul. ${ }^{18}$

$\mathrm{O} \mathrm{pH}$ dos frutos (Tabela 1) apresentou diferenças significativas entre as diferentes espécies, variando de 3,10 a 5,44. Valores semelhantes de $\mathrm{pH}$ foram reportados para butiá $(3,05),{ }^{18}$ araçá vermelho $(3,29),{ }^{13}$ jabuticaba $(2,91-3,72),{ }^{22}$ 
pitanga $(3,27),{ }^{8}$ açaí $(5,6)$, araçá amarelo 'Sabine' $(4,3),{ }^{17}$ jabuticaba $(2,91-3,72)^{22} \mathrm{e}$ guabiroba $(3,47),{ }^{19}$ corroborando os resultados obtidos neste trabalho.

Entre os frutos analisados, somente os de pitanga apresentam na literatura valores referência, os quais atendem aos padrões de identidade e qualidade legais vigentes para AT de no mínimo $0,92 \%$ e pH de 2,5 a 3,4.26

Tabela 1 - Atributos de sólidos solúveis (SS; \%), acidez titulável (AT; \% de ácido cítrico), relação SS/AT e pH em frutos de diferentes espécies nativas da região Sul do Brasil colhidos no ponto de consumo"

\begin{tabular}{lrrrr}
\hline Espécie & SS (\%) & AT (\%) & Relação SS/AT & pH \\
\hline Açaí & $10,22 \mathrm{a}$ & $1,63 \mathrm{a}$ & $6,28 \mathrm{a}$ & $5,44 \mathrm{a}$ \\
Araçá amarelo & $7,06 \mathrm{~b}$ & $1,11 \mathrm{~b}$ & $6,36 \mathrm{a}$ & $3,88 \mathrm{c}$ \\
Araçá vermelho & $7,28 \mathrm{~b}$ & $0,97 \mathrm{~b}$ & $7,50 \mathrm{a}$ & $3,97 \mathrm{~b}$ \\
Butiá & $9,67 \mathrm{a}$ & $1,56 \mathrm{a}$ & $6,20 \mathrm{a}$ & $3,10 \mathrm{f}$ \\
Guabiroba & $10,54 \mathrm{a}$ & $1,80 \mathrm{a}$ & $5,85 \mathrm{a}$ & $3,24 \mathrm{e}$ \\
Jabuticaba & $8,56 \mathrm{~b}$ & $1,52 \mathrm{a}$ & $6,65 \mathrm{a}$ & $3,40 \mathrm{~d}$ \\
Pitanga & $9,02 \mathrm{a}$ & $1,20 \mathrm{~b}$ & $7,51 \mathrm{a}$ & $3,15 \mathrm{f}$ \\
\hline Média & 8,91 & 1,40 & 6,62 & 3,74 \\
CV (\%) & 6,39 & 10,16 & 12,11 & 1,08 \\
\hline
\end{tabular}

Os conteúdos de vitamina $\mathrm{C}$ apresentaram diferenças significativas entre os frutos das diferentes espécies analisadas com valores médios de $56,14 \mathrm{mg}_{100 \mathrm{~g}^{-1}}$ de MF (Tabela 2), com destaque para pitanga, que apresentou os maiores conteúdos

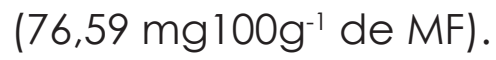

Os conteúdos de vitamina $\mathrm{C}$ apontados na Tabela Brasileira de Composição de Alimentos (TACO) ${ }^{27}$ são de 24,9 e 16,2 mg $100 \mathrm{~g}^{-1}$ de MF para frutos de pitanga e jabuticaba, respectivamente, valores inferiores aos 76,59 (pitanga) e 62,47 (jabuticaba) $\mathrm{mg} 100 \mathrm{~g}^{-1}$ de MF apresentados neste trabalho. Valores inferiores de vitamina $C$ também foram reportados em frutos de jabuticaba coletados em áreas de mata Atlântica, com 13,3 e $25 \mathrm{mgl00}^{-1}$ de $\mathrm{MF}$, respectivamente. ${ }^{2,25}$ No

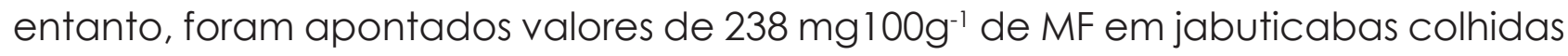
no Ceará. ${ }^{12}$

Os frutos de guabiroba e açaí apresentaram os menores conteúdos de vitamina $C\left(40,65\right.$ e $44,97 \mathrm{mg}^{100 \mathrm{~g}^{-1}}$ de $\mathrm{MF}$, respectivamente). Para guabiroba, os valores são inferiores aos $74,44 \mathrm{mgl00g}^{-1}$ de MF apresentados em frutos de C. leneatifolia coletados no Ceará ${ }^{19}$ e aos $61,5 \mathrm{mg}^{100 \mathrm{~g}^{-1}}$ de MF em frutos de C. adamantium coletados no cerrado brasileiro.' Ainda em frutos de guabiroba colhidos no cerrado, foram reportados conteúdos de $30,58 \mathrm{mg} \mathrm{g}^{-1}$ de massa seca. ${ }^{2}$ Assim como para guabiroba, em frutos de açaí, os conteúdos obtidos neste estudo

\footnotetext{
"Valores seguidos da mesma letra nas colunas não diferem entre si pelo teste de Tukey a $5 \%$ de probabilidade.
} 
também são inferiores aos $84 \mathrm{mg}^{100 \mathrm{~g}^{-1}}$ de MF reportados em frutos de E. oleracea provenientes do Estado do Ceará. ${ }^{12}$

Para butiá, os conteúdos de vitamina C $\left(44,97 \mathrm{mgl00g}^{-1}\right.$ de MF) foram superiores aos 32 e $9,35 \mathrm{mgl00g}^{-1}$ de MF reportados em frutos colhidos no Rio Grande do Sul, ${ }^{5,6}$ porém inferiores os $53 \mathrm{mg} 100 \mathrm{~g}^{-1}$ de MF de frutos coletados em Minas Gerais. ${ }^{28}$

Entre os frutos de araçá, o amarelo apresentou conteúdos de vitamina C superiores aos do vermelho, com valores de 72,25 e 51,13 $\mathrm{mg}^{100 \mathrm{~g}^{-1}}$ de MF, respectivamente. Esses resultados diferem dos obtidos em trabalho conduzido no Rio Grande do Sul, onde o araçá vermelho apresentou conteúdos superiores ao amarelo. ${ }^{30}$ No entanto, os valores são semelhantes aos $62 \mathrm{mg} 100 \mathrm{~g}^{-1}$ de MF reportados para frutos de araçá amarelo ${ }^{30}$ e inferiores aos $174,1 \mathrm{mg} 100 \mathrm{~g}^{-1}$ de MF para araçá vermelho. ${ }^{31}$

Tabela 2 - Conteúdo de vitamina $\mathrm{C}\left(\mathrm{mg} 100 \mathrm{~g}^{-1}\right.$ de MF) em frutos de diferentes espécies nativas da região Sul do Brasil, colhido no ponto de consumo**

\begin{tabular}{lr}
\hline Espécie & Vitamina C \\
\hline Açaí & $44,97 \mathrm{e}$ \\
Araçá amarelo & $72,25 \mathrm{~b}$ \\
Araçá vermelho & $51,13 \mathrm{~d}$ \\
Butiá & $44,97 \mathrm{e}$ \\
Guabiroba & $40,65 \mathrm{f}$ \\
Jabuticaba & $62,47 \mathrm{c}$ \\
Pitanga & $76,59 \mathrm{a}$ \\
Média & 56,14 \\
\hline CV (\%) & 3,28 \\
\hline
\end{tabular}

Os conteúdos de vitamina $\mathrm{C}$ presentes nos frutos avaliados, especialmente em pitanga, araçá amarelo e jabuticaba, são semelhantes e até superiores a outros frutos considerados fontes importantes dessa vitamina, como laranja (Citrus

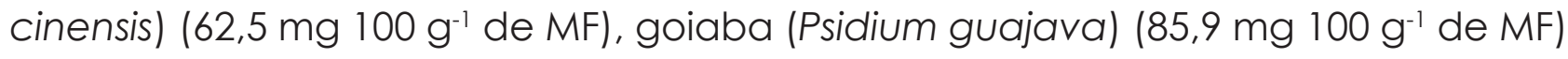
e mamão papaia (Carica papaya) $\left(80,2 \mathrm{mg} 100 \mathrm{~g}^{-1}\right.$ de MF). ${ }^{32,33}$

Para alguns frutos nativos também são reportados valores semelhantes de vitamina C, como goiaba-serrana (Acca sellowiana), com 77,3 e 55,2 $\mathrm{mg}^{100 \mathrm{~g}^{-1}}$ de MF na casca e polpa, respectivamente, e chichá (Sterculia striata), com 89,3 mg $100 \mathrm{~g}^{-1}$ de MF. ${ }^{34,35}$ Porém, outros frutos nativos apresentaram valores superiores de vitamina C, como cajuí (Anacardium humile), cagaita (Eugenia dysenterica), jabotá-do-cerrado (Hymenaea stigonocarpa), macaúba (Acrocomia aculeata) e

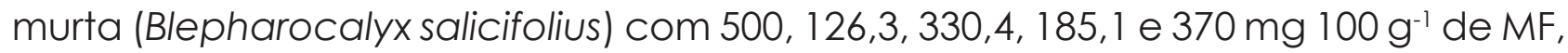
respectivamente. ${ }^{19,35} \mathrm{Em}$ outro trabalho foram reportados valores bem menores de

\footnotetext{
** Valores seguidos da mesma letra nas colunas não diferem entre si pelo teste de Tukey a $5 \%$ de probabilidade.
} 
vitamina C para cagaita (10,63 mg $100 \mathrm{~g}^{-1}$ de MF) e cajú-do-cerrado (Anacardium

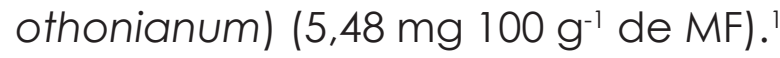

Além da variabilidade genética, o crescimento dos frutos em diferentes condições edafoclimáticas, bem como a época de colheita, o estágio de maturação, o manuseio pós-colheita, o armazenamento e o processamento podem influenciar os teores de vitamina $\mathrm{C}$ dos frutos. ${ }^{5}$

A vitamina $C$ é importante na prevenção do escorbuto e na manutenção da saúde da pele, gengivas e vasos sanguíneos. Também é reconhecido por ter contribuição biológica na formação de colágeno, absorção do ferro inorgânico, redução do nível de colesterol no plasma, redução na formação de nitrosamina, melhora do sistema imunológico humano e grande capacidade de reagir com radicais livres, sendo considerado de alta capacidade antioxidante. ${ }^{36}$ No entanto, a vitamina $C$ não é produzida pelo organismo humano, necessita ser ingerida, tendo as frutas e hortaliças como suas principais fontes. ${ }^{36}$

A ingestão diária recomendada (IDR), estabelecida no Brasil para adultos, é de $45 \mathrm{mg}^{37} \mathrm{e}$, dessa forma, a ingestão diária de $100 \mathrm{~g}$ de frutos de pitanga, jabuticaba, araçá amarelo e vermelho supre totalmente a recomendação de IDR de vitamina $C$.

Apesar de os frutos de guabiroba, açaí e butiá apresentarem conteúdos inferiores, o consumo de $100 \mathrm{~g}$ supre em mais de $90 \%$ da IDR de vitamina $\mathrm{C}$, indicando que são fontes importantes dessa vitamina na dieta humana.

\section{CONCLUSÃO}

Os dados obtidos no presente trabalho indicam que todos os frutos estudados (pitanga, açaí, jabuticaba, araçá, guabiroba e butiá) são fontes importantes de vitamina $C$, destacando-se a pitanga, que apresentou os maiores conteúdos. Além disso, apresentam características físico-químicas adequadas para consumo tanto in natura quanto na forma processada, podendo a utilização desses frutos oferecer enormes oportunidades para a indústria de alimentos convencionais e funcionais.

\section{Agradecimentos}

Os autores agradecem à Fundação de Amparo à Pesquisa e Inovação do Estado de Santa Catarina (FapesC) pelo apoio financeiro, e ao Instituto federal Catarinense (IFC) de Rio do Sul, pela infraestrutura na realização da pesquisa. 


\section{REFERÊNCIAS}

1. Alves AM, Dias T, Hassimotto NMA, Naves MMV. Ascorbic acid and phenolic content, antioxidant capacity and flavonoids composition of Brazilian Savannah native fruits. J. Food Sci. Technol. 2017; 37(4):564-9. doi:10.1590/1678-457X.26716

2. Rosso VV. Bioactivities of Brazilian fruits and the antioxidant potential of tropical biome. Food and Public Health. 2013; 1 (3):37-51. doi:10.5923/j.fph.20130301.05

3. Wu SB, Long C, Kennelly E. Phytochemistry and health benefits of jabuticaba, an emerging fruit crop from Brazil. Food Res Int. 2013; 54(1):148-59. doi:10.1016/j. foodres.2013.06.021

4. Oliveira VB, Yamada LT, Fagg CW, Brandão MGL. Native foods from Brazilian biodiversity as a source of bioactivity compounds. Food Res Int. 2012; 48(1):1709. doi:10.1016/j.foodres.2012.03.011

5. Denardin CC et al. Antioxidant capacity and bioactive compounds of four Brazilian native fruits. J Food Drug Anal. 2015; 23(3):387-98. doi:10.1016/j. jfda.2015.01.006

6. Pereira MC et al. Characterization, bioactive compounds and antioxidant potential of three Brazilian fruits. J Food Drug Anal. 2013; 29(1):19-24. doi:10.1016/j.jfca.2012.07.013

7. Coradin L, Simisnski A, Reis A. Espécies nativas da flora brasileira de valor econômico atual ou potencial: Plantas para o futuro - Região Sul. Braślia: MMA; 2011.

8. Lopes AS, Mattietto RA, Menezes HC. Estabilidade da polpa de pitanga sob congelamento. Ciência Tecnologia de Alimentos. 2005;25(3):553-9. 
9. Campos RP, Hiane PA, Ramos MIL, Ramos Filho MM, Macedo MLR. Conservação pós-colheita de guavira (Campomanesia sp.). Rev Bras Fruticultura. 2012; 34(1):41-9. doi:10.1590/S0100-29452012000100008

10. Schotsmans WC, East A, Thorp G, Woolf AB. Feijoa (Acca sellowiana [Berg.] Burret). In Yahia EM, editor. Postharvest biology and technology of tropical and subtropical fruits: cocoa to mango. Cambridge: Woodhead Publishing; 2011 . p. 115-133.

11. Costa GNS, Mendes MF, Araújo IO, Pereira CSS. Desenvolvimento de um iogurte sabor juçaí (Euterpe edulis Martius): avaliação físico-química e sensorial. TECCEN. 2012;5(2):43-58.

12. Rufino MSM, Alves RE, Brito ES, Pérez-Jiménez J, Saura-Calixto F, Mancini-Filho J. Bioactive compounds and antioxidant capacities of 18 non-traditional tropical fruits from Brazil. Food Chem. 2010; 121 (1):996-1002. doi:10.1016/j. foodchem.2010.01.037

13. Santos MS, Petkowicz CLO, Pereira Netto AB, Wosiack G, Nogueira A, Carneiro EBB. Propriedades reológicas de doce em massa de araçá vermelho (Psidium cattleianum Sabiene). Rev Bras Tecnol Agroind. 2007; 1 (2):104-16. doi:10.3895/ S1981-36862007000200011

14. Instituto Adolfo Lutz. Métodos físico-químicos para análise de alimentos. São Paulo; 2008.

15. Strohecker R, Henning HM. Análises de vitaminas: métodos comprovados. Madrid: Paz Montolvo; 1967.

16. Canteri MG, Althaus RA, Virgens Filho JS, Giglioti EA, Godoy CV. SASM - Agri: Sistema para análise e separação de médias em experimentos agrícolas pelos métodos Scoft - Knott, Tukey e Duncan. Rev Bras Agrocomputação. 201 1; $1(2): 18-24$. 
17. Silva NA, Rodrigues E, Mercadante AZ, Rosso VV. Phenolic compounds and carotenoids from four fruits native from the Brazilian Atlantic forest. J Agric Food Chem. 2014; 62(22):5072-84.

18. Schwartz E, Fachinello JC, Barbieri RL, Silva JB. Avaliações de populações de Butia capitata de Santa Vitória do Palmar. Rev Bras Fruticultura. 2010; 32(2):73645. doi:10.1021/jf501211p

19. Lima JSS, Castro JMC, Sabino LBS, Torres LBV. Physicochemical properties of guabiroba (Campomanesia leneatifolia) and myrtle (Blepharocalyx salicifolius) native to the mountainous region of Ibiapaba-CE, Brazil. Rev Caatinga. 2016; 29(3):753-7.

20. Melo APC, Selequini A, Veloso VRS. Caracterização física e química de frutos de araçá (Psidium guineense Swartz). Comunicata Scientiae. 2013; 4(1):91-5.

21. Amarante CVT, Argenta LC, Basso C, Suzuki A. Composição mineral de maçãs 'Gala' e 'Fuji' produzidas no sul do Brasil. Pesq. Agropec. Bras. 2012;47(4):550-60. doi:10.1590/S0100-204X2012000400011

22. Oliveira AL, Brunini MA, Salandini CAR, Bazzo FR. Caracterização tecnológica de jabuticabas 'Sabará' provenientes de diferentes regiões de cultivo. Rev Bras Fruticultura. 2003; 25(3):397-400. doi:org/10.1590/S0100-29452003000300009

23. Gordon A et al. Chemical characterization and evaluation of antioxidant properties of açaí fruits (Euterpe oleraceae Mart.) during repining. Food Chemistry. 2012; (2):256-63._doi:10.1016/j.foodchem.2011.11.150

24. Chitarra MIF, Chitarra AB. Pós-colheita de frutos e hortaliças: fisiologia e manuseio. Lavras: FAEPE; 2005. 
25. Brunini MA, Oliveira AL, Salandini CAR, Bazzo FR. Influência da embalagem e temperatura no armazenamento de jabuticabas (Myrciaria jabuticaba (Vell) Berg) cv "Sabará". Ciênc Tecnol Aliment. 2004; 24(3):378-83. doi:10.1590/S010120612004000300013

26. Ministério da Agricultura e do Abastecimento (BR). Instrução Normativa n.01 de 07 de janeiro de 2000. Aprova o regulamento técnico geral para fixação dos padrões de identidade e qualidade para polpa de frutas. Diário Oficial da União, Poder executivo. Brasília, DF: Ministério da Agricultura e do Abastecimento; 2000.

27. Tabela Brasileira de Composição de Alimentos. Campinas: NEPA/UNICAMP; 2011.

28. Faria JP, Almeida F, Silva LCR, Vieira RF. Caracterização da polpa do coquinho-azedo (Butia capitata var. capitata). Rev Bras Fruticultura. 2008; 30(3):827-9. doi:10.1590/S0100-29452008000300045

29. Giacobbo CL, Zanuzo M, Chim J, Fachinello JC. Avaliação do teor de vitamina $C$ em diferentes grupos de araçá-comum. Rev Bras Agrociência. 2008; 14(1):155-9. doi:10.18539/cast.v14i1.1899

30. Donadio LC, Moro FV, Servidone AA. Frutas brasileiras. São Paulo: Novos Talentos; 2004.

31. Barcia MT, Jacques AC, Pertuzatti PB, Zambiazi, R. C. Determinação de ácido ascórbico e tocoferóis em frutas por CLAE. Ciênc Alimen. 2010; 31 (2):381-90.

32. Couto MAL, Canniatti-Brazaca SG. Quantificação de vitamina C e capacidade antioxidante de variedades cítricas. Ciênc Tecnol Alimen. 2010; 30(1):15-9. doi:10.1590/S0101-20612010000500003 
33. Oliveira DS, Aquino PP, Ribeiro SMR, Proença RPC, Pinheiro-Sant'ana HM. Vitamina $C$, carotenoides, fenólicos totais e atividade antioxidante de goiaba, manga e mamão procedentes da Ceasa do estado de Minas Gerais. Acta Sci., Health Sci. 2011; 33(1):89-98. Disponível em: doi:10.4025/actascihealthsci. v33i1.8052

34. Amarante CVT, Souza AG, Benincá TDT, Steffens CA. Fruit quality of Brazilian genotypes of feijoa at harvest and after storage. Pesq Agropec Bras. 2017; 52(9):734-42. doi:10.1590/s0100-204×2017000900005

35. Rocha MS, Figueiredo RW, Araújo MAM, Moreira-Araújo RSR. Caracterização físico-química e atividade antioxidante (in vitro) de frutos do cerrado Piauiense. Rev Bras Fruticultura. 2013; 35(4):933-41. doi:10.1590/S0100-29452013000400003

36. Lee SK, Kader AA. Preharvest and postharvest factor influencing vitamin C content of horticultural crops. Postharvest Biol Technol. 2000; 20(3):207-20. doi:10.1016/S0925-5214(00)00133-2

37. Agência Nacional de Vigilância Sanitária (BR). Resolução RDC n 269, de 22 de setembro de 2005. Aprova o regulamento técnico sobre a ingestão diária recomendada (IDR) de proteína, vitaminas e minerais. Diário Oficial da União, Poder Executivo. Brasília, DF: Ministério da Saúde; 2005.

Data de submissão: 02 de março de 2018 Avaliado em: 03 de abril de 2018 (AVALIADOR A) Avaliado em: 10 de abril de 2018 (AVALIADOR B) Aceito em: 29 de abril de 2018 